\title{
LA CONFERENCIA INTERNACIONAL DE POBLACIÓN. MÉXICO, AGOSTO DE 1984*
}

\author{
RAÚL BENÍTEZ ZENTENO**
}

\section{ANTES DE LA CONFER ENCIA}

EL 25 DE NOVIEMBRE DE 1981 el Consejo Económico y Social de las Naciones Unidas, decidió "que la Conferencia Internacional de Población se dedicara a cuestiones determinadas de máxima prioridad, con pleno reconocimiento de las relaciones que existian entre la población y el desarrollo social y económico con el objeto de contribuir al proceso de examen y evaluación del Plan de Acción Mundial sobre Población 1 y a su ulterior ejecución. El Consejo también decidió que la Conferencia se realizara dentro del marco del Plan de Acción Mundial sobre Población existente, cuyos principios y objetivos continuaban siendo plenamente válidos".2

* Publicado también sin el ANEXO en Enlace núm. 11. Boletín Informativo del Programa Regional de la UNESCO sobre Educación en Población. Diciembre de 1984 .

** Investigador del Instituto de Investigaciones Sociales de la UNAM y Secretario Ejecutivo del Consejo Mexicano de Ciencias Sociales.

1 Informe de la Conferencia Mundial de la Población de las Naciones Unidas, Bucarest, 19 a 30 de agosto de 1974. (Publicación de las Naciones Unidas, núm. de Venta: S.75. XIII.3) Subrayado mío.

2 Conferencia Internacional de Población. México, D.F., agosto de 1984. Documento E/CONF.76/BP/1. 6 de junio de 1984. Naciones Unidas. 
La Conferencia se llevó a cabo del 5 al 14 de agosto de 1984 en la ciudad de México, y se constituyó en la culminación de muy diversas acciones preparatorias de cobertura mundial y regional en las que participaron las comisiones regionales (Asia y el Pacífico, Europa, América Latina, África, Asia Occidental y los Estados Árabes);grupos de expertos (sobre los temas: la fecundidad y la familia; la distribución de la población, la migración y el desarrollo; población recursos medio ambiente y desarrollo; y la mortalidad y la política sanitaria); y el Comité preparatorio que en dos reuniones pudo elaborar un texto con 85 recomendaciones a la Conferencia para la ulterior ejecución del Plan de Acción Mundial sobre Población. 3

Todo lo anterior permitió que en la Conferencia, a la que asistieron representaciones de 146 paises, * se pudiese llegar a un acuerdo, cuyas resoluciones quedaron plasmadas en 88 recomendaciones aprobadas por consenso con formulación de reservas para tres de ellas. ${ }^{4}$

La organización del evento permitió llegar a la aprobación del informe sin que se produjeran debates con posiciones irreductibles o enfrentamientos que obligasen a ampliar la Conferencia un día más como se tenía previsto para tal contingencia. Lo anterior se debió, entre otros aspectos a: 1) todo el proceso preparatorio a que ha hecho mención; 2) a la acumulación de experiencias sobre los diez años transcurridos desde la reunión de Bucarest; 5 3) a la aceptación de la vigencia del Plan de Acción Munđial, de sus avances y logros y 4) a la existencia de una posición generalizada que aconsejó no reabrir el debate de Bucarest y continuar con lo que se inició entonces. Aquí conviene hacer algunas consideraciones para cada una de las cuatro afirmaciones anteriores:

\section{Aspectos del proceso preparatorio de la Conferencia.}

El Proceso preparatorio de la Conferencia hizo posible partici-

3 Recomendaciones para la ulterior ejecución del Plan de Acción Mundial sobre población. E/CONF.76.5. 6 de junio de 1984. Naciones Unidas.

* También observadores de 3 organizaciones o movimientos de liberación nacional; funcionarios de la Secretaría de 3 oficinas de Naciones Unidas; 4 Comisiones Regionales de N.U.; 5 organizaciones especializadas; 13 organismos intergubernamentales y representantes de organizaciones académicas.

4 Aprobación del Informe de la Conferencia. Informe de la Comisión Principal. E/CONF.76/L.3.13 de agosto de 1984. Naciones Unidas.

5 Resumidas en el documento: Examen y Evaluación del Plan de Acción Mundial sobre Población. Informe del Secretario General. E/CONF. 76/4, 19 de junio de 1984. Naciones Unidas. 
BENITEZ: LA CONFERENCIA INTERNACIONAL

paciones previas de los Gobiernos y arribar a la reunión con un buen conocimiento de lo que allí se trataría.

Por otra parte, se contó con el informe del examen y evaluación del Plan de Acción Mundial resultado de los debates del Comité Preparatorio de la Conferencia ${ }^{6}$ en el que se expone el fundamento y las bases de las propuestas relativas a las recomendaciones para la ulterior aplicación del Plan de Acción. Este documento facilitó las deliberaciones de la Conferencia de los seis temas que trata: desarrollo socioeconómico y Población: desarrollo de las políticas de población; tendencias perspectivas, metas y políticas de población; promoción del conocimiento; la función de los gobiernos nacionales y de la comunidad internacional; y observación, examen y evaluación del Plan de Acción Mundial sobre Población.

$\mathrm{El}$ informe se basó en los resultados de las cuatro reuniones de expertos convocados como parte de los trabajos preparatorios, la observación de las tendencias y políticas en material de población y las conclusiones de la Quinta Encuesta Demográfica entre los Gobiernos y fue preparado por la División de Población del Departamento de Asuntos Económicos y Sociales Internacionales en cooperación con el Departamento de Cooperación Técnica para el Desarrollo, el Fondo de las Naciones Unidas para actividades en Materia de Población, las comisiones regionales, los organismos especializados y otros órganos del sistema de las Naciones Unidas, así como también diversas organizaciones no gubernamentales.

\section{La acumulación de experiencias.}

La evaluación del Plan de Acción diez años después implicó la necesidad de tener información que mostrase los avances a través de la acumulación de experiencias. El tema obligado es la manera en que se ha dado el desarrollo de políticas de población y evaluación de su eficacia, las cuales deben integrarse en los planes y programas sociales y económicos. En este aspecto se reconoce lo mucho que falta por hacer y si bien se acepta el principio de la integración, en la práctica se sigue considerando a la población de manera independiente y sólo a partir de proyecciones demográficas.* Los problemas que aparecen se rela-

6 Examen y Evaluación del Plan de Acción Mundial. Informe del Secretario General. Naciones Unidas E/CONF./76.4.

* Las proyecciones estimadas a partir del comportamiento histórico de la fecundidad, la mortalidad y las migraciones, sin tomar en cuenta los efectos, sobre estos factores, de los cambios económicos y sociales. Es aquí en donde se carece aún de elementos que establezcan la relación entre dichos cambios económicos y sociales como consecuencia del desarrollo y la población. 
cionan con la carencia de una teoría que sea manejable en los distintos niveles de operación de la acción planificadora y útil para la programación de acciones concretas en las distintas áreas de operación gubernamental y que además coordine o tome en cuenta programas de grupos o sectores privados. Todo lo anterior implica enfrentar la viabilidad política y social de lo que se establece al nivel de la política demográfica. De allí que los progresos han sido lentos sobre todo en la formulación de fundamentos teóricos fiables y viables para integrar las políticas y programas de población en las políticas nacionales de desarrollo. La implementación de lo anterior se ha limitado por las dificultades para la obtención y uso de la información necesaria sobre las interrelaciones demográficas, con las sociales y económicas. La consideración de estos aspectos en el informe se señalan junto con otros más específicos como el avance en la creación de dependencias que traten los aspectos demográficos $\mathrm{y}$ aporten elementos para la integración.

Otro tema necesario para poder hablar de la acumulación de experiencias en la Conferencia es la evaluación de los avances como un elemento fundamental que sirva a las finalidades políticas. El informe hace una detallada mención de la complejidad de esta tarea sobre todo en temas como los de migración o en la búsqueda de medición de los efectos de algún factor relevante que trae cambios demográficos, por ejemplo los efectos de la educación en la reducción de la mortalidad o frente a la planificación familiar. Aparecen también la falta de recursos y de personal calificado, aspectos que son más difíciles de resolver cuando se enfrenta la necesidad de solución de problemas teóricos, en particular en relación con ámbitos geográficos reducidos o de grupos sociales en cuyo caso hay necesidad de proyectos de investigación para la evaluación complejos.

No podía faltar para la Conferencia la información sobre tendencias y perspectivas de la población en relación con politicas demográficas. Del decenio que va de 1970-1975 a 19801985 la tasa de crecimiento anual mundial pasó de 2.0 a 1.7 por ciento. Si se elimina a China de los países en desarrollo tal disminución apenas ha sido significativa (de 2.5 a 2.4 por ciento, sobre todo por el hecho de que la baja de la mortalidad compensó al $15 \%$ de menor fecundidad. Tal tasa de crecimiento mundial corresponde a la estimada en 1974 en el Plan de Acción para el año de 1985 como variante baja, es decir se llegó al $1.7 \%$ de crecimiento medio anual un poco antes de lo previsto. Sin embargo no se llegó a esta cifra de la manera en que se planteó en las proyecciones. Por ejemplo en lugar de disminuir la tasa de crecimiento demográfico para África se ha elevado de 2.7 a 3.0 por ciento por año, debido a la lenta disminución de 
la mortalidad y al mantenimiento de la natalidad en sus elevados niveles, como sucedió en América Latina sobre todo de 1950 a 1960. Para América Latina el cambio fue más lento del esperado, pasó de 2.5 a 2.3 por ciento. En las situaciones asiáticas se dio mayor heterogeneidad. Para Asia Oriental la reducción de la tasa de crecimiento a más de la mitad en diez años en que pasó de 2.3 a 1.1 por ciento (como resultado de la reducción del crecimiento en China y República de Corea), difiere de la de Asia Meridional o Asia Sudoriental y Sudcentral con reducción más pequeña o sin cambios como Asia sudoccidental.

En cuanto a la mortalidad en África no se llegó en 17 países a una esperanza de vida de 50 años como se deseó como mínimo para cualquier país, y otros tantos no disminuyeron la mortalidad infantil por debajo de 120 por mil nacidos vivos. En los países asiáticos la mortalidad se da en un nivel intermedio entre África y América Latina y con mayor diversidad que en estas regiones, desde una esperanza de vida al nacimiento de 37 años en Afganistán a 74 en Hong Kong o Israel. Para América Latina se esperó arribar a una esperanza de vida de 62 años en 1985 y se llegó a 64 años en el periodo 1980-1985. Los países desarrollados lograron en 1983 una esperanza de vida de 73 años, con 77 años para Japón, seguido de América del Norte, y los niveles más bajos se observan en Europa oriental con 71 años.

En cuanto a fecundidad las recomendaciones del Plan de Acción Mundial descartaron el establecimiento de normas al tamaño de la familia o a la diversidad de objetivos nacionales con respecto a las variables que tienen incidencia en los cambios en la natalidad, a partir del respeto del derecho de las personas a decidir de manera libre, informada y responsable, el número y espaciamiento de sus hijos. Lo anterior corresponde a las variaciones que se observan en la fecundidad entre los países en desarrollo; esto último debido a la persistencia de tasas elevadas de fecundidad en gran parte de África y Asia Occidental y la disminución observada en algunos países de Asia y América Latina. En los diez últimos años la disminución de la fecundidad en los países desarrollados ha sido menor que en años anteriores y en algunos casos se han dado pequeños aumentos.

En cuanto a la tasa global de fecundiad se estima para África en 6.4 por mujer en 1980-1985, mientras que diez años antes fue de 6.5; para América Latina la disminución para el mismo periodo fue de $18 \%$ (de 5.0 a 4.1 hijos por mujer); para Asia, debido a la disminución en China, la reducción fue de $51 \%$, pasando de 4.5 a 2.3 hijos por mujer. En Asia Meridional fue de 5.7 a 4.6 .

El informe en que aparecen los datos anteriores (que han 
sido muy extractados para los fines de este escrito) y que permitió a la Conferencia la evaluación del Plan de Acción Mundial, dio una visión completa de los điez años transcurridos tanto de las situaciones demográficas como de su asociación con la gama de factores sociales, económicos y políticos intervinientes, así como de las prácticas de planificación de la familia y el acceso a los servicios; las percepciones, objetivos y metas de los gobiernos; sobre información y educación; políticas económicas; condición de la mujer; sobre distribución de la población y migración interna; la migración internacional; los derechos humanos; la estructura de la población; promoción del conocimiento; así como los diversos aspectos de estos temas en desgloses particularizados que dan cuenta de la situación mundial, por regiones y también por países.

Especial atención se dio a los aspectos relacionados con las acciones orientadas a la manera en que se lleva a cabo la integración de las políticas demográficas en las políticas socioeconómicas en donde falta mucho por hacer.

\section{DURANTE LA CONFERENCIA}

3. La aceptación de la vigencia del Plan de Acción, de sus avances y logros

E1 desarrollo de la Conferencia estuvo precedido también por las declaraciones de 92 Jefes de Estado y de Gobierno ${ }^{7}$ que expusieron brevemente sus opiniones sobre las cuestiones de población, a petición del Secretario General de la Conferencia y para conocimiento de los participantes. Lo anterior no significó que las representaciones siguieran con gran apego a las orientaciones previas, sino que también se dio un cierto libre juego de posiciones con fines más de política muy inmediata que de evaluación del Plan de Acción Mundial. No faltaron las presiones políticas y declaraciones contrarias de "rechazo a toda confrontación ideológica que interfiera en la solución de los problemas que, como el de población afectan a la humanidad y están por encima de las divergencias políticas"; 8 así como la insistencia en el carácter técnico de la Conferencia; el rechazo del condicionamiento de ayuda a los países en desarrollo por divergencias políticas e ideológicas empleando temas como el

7 Perspectivas en materia de población. Declaraciones de los dirigentes del mundo. Fondo de Naciones Unidas para Actividades de Población. Nueva York, junio de 1984. ISBN:0-89714-032-X.

8 Declaraciones de Javier Pérez de Cuéllar a su arribo a México para clausu. rar la Conferencia. Novedades 13 de agosto de 1984. 
de la población; la oposición a la implementación de esquemas sobre control natal como el aborto y la esterilización. Desde luego no faltaron las noticias radicales basadas en declaraciones de la conferencia de Provida y Profamilia que se llevó a cabo casi paralelamente a la Conferencia Internacional, denunciando a 20000 familias norteamericanas multimillonarias de que promueven en todo el mundo los métodos de anticoncepción artificial o que se llega hasta 40 millones de abortos en los países en donde el aborto es ilegal. ${ }^{9}$

\section{Exposiciones $y$ debates}

Addemás de las anteriores posiciones conviene brevemente exponer el desarrollo de algunos debates con mayor detalle, lo que permitirá tener una visión de la manera en que se desarrolló la Conferencia y los problemas tratados, teniendo en cuenta, tal y como se indicó al inicio, que se llegó a un amplio consenso y que el gran esfuerzo de evaluación y reorientación del Plan de Acción Mundial constituye un nuevo avance en la consideración de los problemas de población en los niveles nacionales y mundial. El hecho sobresaliente fue el mantenimiento de los principios y aceptación de los avances y limitaciones del Plan de Acción Mundial como marco de referencia de toda la reunión. Desde su inicio en la Conferencia se hizo mención al principio básico del derecho soberano de que cada nación determine su política poblacional; la importancia de la reunión para las naciones como un esfuerzo continuo por mejorar la calidad de vida de sus pueblos; las dificultades económicas y la crisis actual, particularmente dolorosos en los países en desarrollo; la necesidad de reducir las injusticias y las desigualdades entre naciones y entre individuos; señalamientos concretos en relación con la lucha de los países en desarrollo, por sanear sus economías, el enfrentamiento a restricciones proteccionistas, a muy elevadas tasas de interés y graves problemas de financiamiento, mientras el servicio extenuante de la deuda externa significa severas limitaciones al mantenimiento del desarrollo social. De igual manera se establecieron posiciones sobre todo en relación con la vinculación de lo demográfico y los factores sociales, culturales, económicos, tecnológicos y científicos (aspecto al que haré referencia después), así como el señalamiento de que el crecimiento económico sólo influye apreciablemente en

9 Nota de Alberto Rocha, reportero. El Universal, lunes 13 de agosto de 1984, sobre la inauguración el 12 de agosto del Congreso Internacional Provida y Pro-familia, del movimiento Protección a la Vida en todas las Naciones. 
las variables demográficas si va acompañado de cambios sociales y de la definición de objetivos al futuro. 10

En las reuniones de trabajo de los días siguientes se hicieron afirmaciones que a la vez que mostraban posiciones nacionales $\mathrm{y}$ visión de los problemas en el ámbito internacional, se constitúan paso a paso en apoyo a los principios y recomendaciones elaboradas por el Comité Preparatorio.

Tesis como las siguientes: "los principios de Bucarest son válidos sin injerencias externas en materia de política demográfica" (Brasil); "la necesidad de una vigorosa cooperación mundial que contribuya a la normalización económica y la preservación de la paz. Sólo así se logratán las condiciones para que las naciones puedan alcanzar una efectiva política de desarrollo de población" (Argentina); el control demográfico por sí mismo no es solución para superar la crisis ni los problemas de desarrollo (expresado de muy diversas formas y reiterado por buena parte de las delegaciones); "una política de población que no respeta la libertad de los individuos pierde la esencia de su sentido (Argentina y otros muchos); "reconocimiento de los derechos de las minorías indigenas" (Australia); "debe reconocerse la interrelación entre la población y el medio ambiente" (diversas delegaciones); varias delegaciones hicieron votos por que se superen los enfoques simplistas que tienden a identificar los problemas de población con una determinada tasa de crecimiento demográfico o de fecundidad.

El Banco Mundial al presentar su informe sobre El Desarrollo Mundial 1984, sostiene sus tesis ya conocidas de que el rápido crecimiento de la población exacerba la dificultad de elegir entre un mayor consumo ahora y las inversiones necesarias para lograr un mejor consumo en el futuro; consideró que el rápido crecimiento de la población es un problema de desarrollo, pues el costo principal de tal aumento, soportado principalmente por los pobres de los países en desarrollo, ha sido y será la pérdida de oportunidades para mejorar el nivel de vida de las personas; afirmó que el crecimiento de la pobla. ción no reviste características dramáticas de una crisis financiera o de un trastorno político, pero tiene la misma importancia en lo que respecta a moldear el mundo de las nuevas generaciones; que los problemas demográficos en la mayoría de los países en vías de desarrollo han surgido también por fallas en las po-

10 De las intervenciones en la ceremonia de inauguración el lunes 6 de agosto: de Rafael M. Salas, Secretario General de la Conferencia, de Bi Jilong Subsecretario de la ONU, y de Miguel de la Madrid Hurtado, Presidente Constitucional de los Estados Unidos Mexicanos. 
líticas de sus gobiernos; y que nadie puede sostener que el aumento mas lento de la población puede garantizar por sí mismo el progreso, pero hay pruebas concluyentes de que en muchos países en desarrollo se postergará indefinidamente el progreso.

La reina de Jordania, Noor Al Hussein apuntó que desgraciadamente son tantos los conflictos que muchos de los recursos financieros se utilizan para alimentar las guerras y no a los pueblos. El mundo gasta más de seis mil millones de dólares anuales en armamento y dos mil millones de dólares en programas de planificación familiar. Denunció que cuando hablamos de poblaciones que han sido devastadas por conflictos políti$\cos$ y que han sido diezmadas por el abuso económico de una potencia ocupante, to que queremos decir son los niños que han crecido sin saber si podrán terminar su escolaridad. Queremos decir familias que no saben cuándo van a confiscar o a expropiar sus terrenos y sus hogares; queremos decir hombres y mujeres incapaces de obtener empleo lucrativo en su propia patria.

A su vez la Conferencia sirvió de tribuna para exponer ante el conjunto mundial representado grandes problemas nacionales, en íntima relación con los conflictos entre paises y áreas de influencia de las potencias. El representante de Kampuchea pidió el auxilio de la comunidad internacional ante el genocidio ejercido por la invasión extranjera que ha reducido la población de 8 a 6 millones de habitantes. El representante de España recalcó la urgencia de que los países se preparen para dar ocupación a los jóvenes que cada dia demandan empleo. Se dieron demandas de Túnez y Yemen para que Israel desaloje los territorios ocupados, a lo cual Israel indicó que no deben salirse de la temática de la Conferencia. Italia dio gran importancia a la trascendencia de la información de los fenómenos demográficos, así como el respeto que debe darse a los derechos de los inmigrantes.

Por su parte el representante vaticano reiteró la posición de la Iglesia en relación con la urgencia de que los gobiernos desarrollen las condiciones para que la población tenga la posibilidad de educarse para decidir libremente el número y espaciamiento de sus hijos, de acuerdo con los métodos naturales. La oposición de la Iglesia católica ante el aborto fue reforzada con el argumento de que la vida humana debe ser respetada desde el momento de la concepción. También se opuso a la esterilización y al uso de anticonceptivos.

Por su parte y en contradicción con los reiterados plantemientos norteamericanos, la delegación presidencial rechazó el catastrofismo y el malthusianismo y se mostró optimista por los logros de los últimos 10 años en la reducción de la tasa de 
natalidad mundial, así como su desacuerdo con la visión pesimista del Banco Mundial. En otros momentos la delegación norteamericana logró la oposición de todos, salvo Israel, al pedir se suprimiera un párrafo en donde la comunidad internacional condena el establecimiento de asentamientos en territorios ocupados por la fuerza. Lo anterior como defensa de la ocupación de Israel en territorios árabes y el señalamiento de que también había asentamientos de vietnamitas en Kampuchea y de soviéticos en Afganistán (no hubo menciones a Inglaterra y las Malvinas). También se rechazó la posición norteamericana en torno a la tesis de la libre empresa como solución a los problemas demográficos. Se señaló por un número importante de delegados que la enmienda norteamericana va en contra del espíritu mismo de la Conferencia que reconoce ante todo que una transformación económica y social en el mundo es el requisito para logar una solución efectiva a los problemas demográficos.

La propuesta quedó inscrita en la recomendación 3 del informe definitivo de la siguiente manera "(...) y alentar diversas fuentes de inversión y, cuando corresponda, las iniciativas empresariales (...)".

Portugal, a través de su ministro de salud dijo que el evento reviste importancia al realizarse en un país cuyo prestigio internacional ha aumentado por la defensa que realiza de la paz y el humanismo, como lo prueba en el presente su papel en el grupo de Contadora, e indicó que un desarrollo integrado a nivel mundial no puede instaurarse sin que se garantice la independencia y la soberanía de las naciones.

Para Polonia el momento actual no es el mas favorable para aplicar la justicia e igualdad en el mundo ya que para encontrar soluciones a los problemas demográficos debemos luchar por la paz y por el desarme especialmente nuclear. El establecimiento de mejoras políticas y sociales puede contribuir al propósito anterior y que en el caso de su país se trabaja para conceder privilegios a la mujer y a los niños.

E1 representante de Malasia enfatizó que debemos tomar el ejemplo de los países de Asía Oriental en cuanto a trabajar por un desarrollo independiente y evitar los hábitos negativos de consumo como los países desarrollados. Debe existir la conciencia de que las naciones ricas otorguen mayores flujos de capital que sirvan a los pobres a sostener recursos necesarios para su desarrollo.

La representación de Yemen, además de la condena política de desafío e invasión de Israel que causan tremendos problemas a la región árabe, indicó que en Bucarest los logros de una estrategia poblacional no llegaron al nivel deseado en aquella 
ocasión y en la actualidad resulta imperante para cada país liberar la relación desarrollo-población, de la dependencia externa.

Costa Rica manifestó que la conflictiva situación que prevalece en Centroamérica ha repercutido en el país en donde en los últimos años la población se ha incrementado en un diez por ciento, e hizo un llamado a todos los países a reducir o eliminar los gastos de armamentismo y que ese dinero se canalice al desarrollo. Recalcó que los problemas poblacionales deben ser resueltos por cada país y rechazó la idea simplista de que el control natal mejora la situación económica, y las medidas coercitivas que controlan el derecho al matrimonio y el de residencia.

Por su parte la delegación china indicó que gracias a su política demográfica se ha logrado abatir el crecimiento de la población a $1.54 \%$ al año, e indicó que los intentos por disminuir el problema de la población deben ir junto con los esfuerzos para establecer un nuevo orden económico internacional. En este mismo sentido se manifestó la delegación de la India y agregó que el Movimiento No Alineado nos provee la única esperanza para un clima duradero de paz internacional, así como de seguridad y que lo contrario nos llevaria a una "raza" totalmente armamentista y a un desvio injustificado de los recursos económicos. La delegación soviética replanteó su tesis sobre la carrera armamentista indicando que con ella se pretende realizar una política de fuerza e interferencia y de monoscabo a la independencia y soberanía de los Estados y a su vez es una de las causas principales de la profundización e inestabilidad política y económica del mundo, que aumenta la amenaza nuclear y pone en peligro la existencia misma de la humanidad. Manifestó que el informe sobre el Plan de Acción Mundial sobre Población tiene un carácter demasiado técnico que refleja deficientemente los factores socioeconómicos de los procesos demográficos en distintas regiones y países y que de hecho no contiene la idea sobre la interrelación entre la población y el desarrollo. Es difícil que alguien se oponga al concepto de que los procesos demográficos están relacionados estrechamente con el desarrollo socioeconómico, pues son una condición fundamental. El documento central de Bucarest no contiene la idea precisa sobre la interacción entre estos dos factores, por lo que es preciso analizar la influencia recíproca de políticas demográficas, sociales, con el desarrollo. De cualquier manera el Plan de Acción Mundial sobre Población no necesita revisión "sigue completamente en vigor".

La delegación cubana hizo mención del hecho de que la Conferencia se produce en un mímento de profunda crisis mundial que se sufre en particular en los paises subdesarrollados como consecuencia de la dependencia externa y la con- 
siguiente distorción estructural de su economía. Cuba, como el mejor aporte de los aspectos más positivos de Bucarest, expone su experiencia, fundamentada en que a pesar del bloqueo estadunidense a su economía, ha superado la mayoría de los problemas. El discurso del delegado cubano provocó la intempestiva salida de varias delegaciones encabezadas por la estadunidense. Desde la tribuna se trasmitió el mensaje de Fidel Castro: "ante la tragedia nuclear y la crisis no cabe la resignación y acomodo, la salida es la lucha incansable por la paz y el desarrollo". El representante búlgaro hizo un paragón entre las décadas de los setentas y de los ochentas. En la primera había distensión y optimismo entre los pueblos del mundo y ahora, en México, la característica es de una situación internacional tensa causada por las fuerzas imperiales, por una carrera armamentista sin precedentes y una crisis económica profunda que afecta a los países más débiles, sin la posibilidad cercana de que puedan superarse los obstáculos que impiden su desarrollo. Sin estos aspectos y sin considerar la importancia de la paz y la cooperación internacional es imposible abordar los temas demográficos.

El encargado del Programa de las Naciones Unidas para el desarrollo recalcó la injusticia implícita en la petición a los países pobres de que no aumenten su población cuando se gastan enormes cantidades en armamentos, se aumentan las tasas de interés y se impide el establecimiento de un orden internacional más justo. Diez años después de Bucarest la situación demográfica mundial sigue siendo preocupante. No se han logrado las condiciones necesarias que aseguren mejores niveles de vida a todos los habitantes del planeta. No se debe calificar a los pobres de delincuentes demográficos, cuando los que más tienen les niegan ayuda. No se debe ver como lacras a los campesinos que llegan a las ciudades, sino como refugiados de una mala distribución del desarrollo. Para resolver esto es necesario responder a un reto por parte de la comunidad internacional. Un reto de solidaridad y ayuda multilateral.

La Organización Mundial de la Salud por su parte recalcó la tesis de que la decisión sobre los hijos no debe corresponder sólo a las mujeres y es por eso que ha intensificado una campaña para activar la participación masculina en el proceso de planificación familiar. También elaborará en breve una inyección aplicable a los hombres que los mantendrá "fuera del mercado por uno, dos o tres años". Se negó que la OMS y las transnacionales estén coludidas para experimentar con nuevos medicamentos de control natal en las naciones en desarrollo. Se reconoció que ha habido debilidad en los sistemas de salud, especialmente en lo relativo a la planificación familiar, que no se ha impartido con la tecnologia adecuada, pues es frecuente que las mujeres 
Benítez: LA CONFERENCIA INTERNACIONAL

que utilizan anticonceptivos se asusten con los efectos secundarios que les ocasionan. Cuando se preguntó al representante si la OMS apoyo el aborto, dijo que no, aunque se ha solicitado auxilio y se pide una política humana, de decencia, de recurso, de responsabilidad ya que 200000 mujeres mueren en abortos ilegales, sin mencionar el daño mental y físico que les causa la intervención. También indicó que los inventos enfocados ala salud como el corazón de plástico dentro de 200 o 300 años se venderán en el supermercado. También demandó el restablecimiento del diálogo de las naciones del norte con las del sur dada la interdependencia que existe entre ellas y terminar con las hambrunas que sufren 800 millones de personas en el mundo.

La Organización de las Naciones Unidas para la Educación, la Ciencia y la Cultura mencionó los diversos campos de actividades y estudios que lleva a cabo, destinados a promover un enfoque interdisciplinario en el análisis de las relaciones entre varios aspectos de los problemas considerados y a promover la aplicación de los resultados obtenidos en la formación de personal nacional para la extensión de los programas. Indicó que se llevan a cabo en muchos países del mundo en desarrollo proyectos de población en materia de educación, de comunicación y de información. Estos proyectos son ejecutados en apoyo a programas de bienestar social y desarrollo de la comunidad a solicitud de los Estados Miembros. Resultó de interés el señalamiento de UNESCO de que el futuro exigirá impostergablemente nuestra atención hacia nuevas situaciones que están emergiendo debido a cambios demográficos, sociales y culturales, como por ejemplo, cambios en las estructuras de la familia y su impacto sobre la situación de las mujeres; las consecuencias para la sociedad del proceso de envejecimiento de la población." En el programa de UNESCO para 1984-1985 se da importancia al mejoramiento de la condición de la mujer, tema al cual se ha dado especial atención en el Organismo. Las actividades incluyen la igualdad de oportunidades educativas a todos los niveles para las niñas y las mujeres; investigaciones sobre el papel de la mujer como agente activo en los procesos sociales y cuturales, la eliminación de todo tipo de discriminación y otros muchos.

La organización de las Naciones Unidas para la infancia y la Familia, culpó a los gobiernos de dejar a los niños en la última instancia, en lugar de la principal prioridad e hizo señala-

* Sobre este tema UNESCO preparó un estudio sobre Educación y envejecimiento. Esta labor fue destacada en el Plan de Acción Internacional de Viena aprobado por la Asamblea Mundial sobre el Envejecimiento, celebrada en 1982. 
mientos sobre la atención que debe darse a la mortalidad infantil, nutrición, servicios básicos, inmunización contra enfermedades, educación de la mujer para el mejor cuidado del infante y la contribución de la planificación familiar para el bienestar maternoinfantil.

Durante la Conferencia se hicieron intervenciones de muy distinta naturaleza y las que han sido mencionadas constituyen un muestrario de algunos de los temas que más llamaron la atención. El conjunto de 88 recomendaciones aprobadas constituye una versión modificada de Bucarest, frente a lo demográfico, necesariamente orientada por ideologías y teorías de la población y el desarrollo que van hacia la pluralidad y en donde sobresalen posturas aceptables para todos dentro del lenguaje internacional inspirado por la idea del consenso y que permite a cada país llevar a cabo la política que convenga.

\section{Posición generalizada: no reabrir el debate de Bucarest}

Las recomendaciones en que se enmarca el Plan de Acción Mundial sobre Población, a partir de la Conferencia de México, tomaron en cuenta situaciones demográficas nuevas y un pragmatismo considerable basado en el planteamiento general de la necesidad de un enfoque integrado que reconoce las interrelaciones entre la población, los recursos, el medio ambiente y el desarrollo; lo anterior orientado a lograr programas de acción en los que se integren precisamente la población y el desarro1lo. De alguna manera lo que se busca es lograr en la práctica lo que se postula en buena parte de las orientaciones de las distintas posiciones teóricas, sobre los condicionantes de los cambios demográficos, con apoyo en gran cantidad de información y análisis, en los que se establecen relaciones, generalmente indirectas, entre los factores demográficos y los sociales y economicos. También se consideró con mayor énfasis la situación internacional sobre todo para el avance de los países atrasados. A su vez se reafirmó que la base para una solución efectiva de los problemas demográficos es "ante todo la transformación económica y social".

Conviene señalar que lo anterior equivale a los planteamientos de los demógrafos latinoamericanos que desde hace 20 años enfrentaron las ideas neomalthusianas, en las que predominó la posición de que el control del crecimiento demográfico haria posibles avances económicos y sociales. En estos análisis no se tomaba en cuenta la situación mundial, o bien se consideraba a los países como entidades cerradas.

Debe mencionarse que en la Conferencia se desestimaron de inmediato las amenazas condicionadoras de la ayuda externa 
a los países pobres en donde se acepte el aborto, o las posiciones oportunistas que emplean los postulados propios como forma de presión sobre problemas internacionales o internos de otros países. En estos casos la participación de la delegación norteamericana fue desafortunada. Tampoco se buscó rediscutir la posición que planteó en Bucarest hablar primero de un nuevo orden económico internacional y después de población. De hecho en México se trató a los dos temas, dando prioridad a la búsqueda de mejores condiciones de vida de la población.

Las situaciones demográficas nuevas o reconocidas ahora, se resumen en la visión más cercana e informada del curso que ha tomado la transición demográfica en los países de desarrollo intermedio, en los atrasados y también el señalamiento de problemas como los de envejecimiento en los países desarrollados.

El pragmatismo con que se actuó en la Conferencia Internacional de Población fue el resultado del conocimiento insuficiente de las relaciones entre la población y el desarrollo sobre todo para poder integrar a la población en los programas gubernamentales. Las experiencias aún son muy pobres y en general el logro de avances implica cambios sustantivos del orden internacional y de las situaciones nacionales. La insuficiente discusión sobre estos aspectos implicó el tácito acuerdo de no reabrir el debate de Bucarest, y dar fuerza a la tarea de evaluación de los diez años transcurridos. De cualquier manera quedaron señalados problemas de gran importancia, como el hecho de que cumplimiento de los Derechos Humanos forma parte de la confrontación Norte-Sur; la presencia de una situación demográfica de muy elevado crecimiento en África; el que la planificación familiar no se ha iniciado en los paises musulmanes; el mayor abandono de posiciones teológicas como telón de fondo de las discusiones; la visión más anticipada del futuro de las poblaciones; la mayor congresión en problemas como los de la migración internacional; la insistencia sobre situaciones bélicas y los problemas de los refugiados y también la especial consideración de la función y condición de la mujer. A su vez no sólo quedó inscrita la relación fundamental entre la paz y el desarrollo, sino que quedó señalada la importancia de asignar recursos a programas sociales y económicos y no a programas militares lo que "contribuiría" en gran medida a logar las metas y objetivos del Plan de Acción Mundial sobre Población.

\section{LOS RESULTADOS DE LA CONFERENCIA \\ INTERNACIONAL DE POBLACIÓN}

La CONFERENCIA CUlminó AL APROBAR la Declaración de la Ciu- 
dad de México sobre Población y Desarrollo11 que propusieron 29 Estados y que buscó sintetizar y dar mayor fuerza a las recomendaciones aprobadas el mismo día, para la continuación de la aplicación del Plan de Acción Mundial sobre Población aprobado en 1974 por 138 gobiernos presentes en la Conferencia Mundial celebrada en $\mathrm{Bu}$ carest. Tal declaración no logró sus objetivos y constituye una versión incompleta del Informe de la Conferencia de México (Documento citado E/CONF. 76/L.3; 13 de agosto, 1984), el cuál, además de las 88 recomendaciones que contiene (ver Anexo) está precedido de un Preámbulo, el que junto con la introducción a cada capítulo de las recomendaciones constituye un documento en el cual queda expresada la visión mundial de la problemática de la población y la necesidad de contemplarla en términos de una visión totalizadora en sí misma y como elemento orientador de la acción de los gobiernos sobre lo demográfico, lo económico y lo social, buscando, en la planeación, la integración de los factores demográficos con los factores del desarrollo sobre los que se actúa. Es decir, la búsqueda para lograr cambios en ambos tipos de factores.

La Conferencia de México tomó en cuenta sobre todo los siguientes principios que guían la acción del Plan Mundial aprobado en $\mathrm{Bu}$ carest: que las metas y políticas de población forman parte integral de los objetivos sociales, económicos y culturales que buscan superar los niveles y calidad de vida de la población (principio primero); que las variables demográficas influyen sobre el desarrollo y a la vez el desarrollo tiene un papel crucial y afecta las tendencias demográficas (principio tercero); que el establecimiento de políticas de población constituye un elemento básico de las de desarrollo pero no las substituyen (principio cuarto): se reconocen los derechos a la vida, a la decisión libre y responsable para tener los hijos que se deseen y cuando se decida y la igualdad de la mujer (principios quinto, sexto y octavo); se establece la protección a la familia (principio séptimo); se reconoce la diversidad de situaciones y condiciones entre los países (principio octavo); se señala que las políticas deben formularse democráticamente (principio décimo); se reconoce que la formulación e implementación de políticas de población es un derecho soberano de cada nación y se da a la cooperación internacional un papel relevante de acuerdo a los diversos principios que rigen los más elevados acuerdos que regulan el orden internacional y los derechos humanos.

11 Declaración de la Ciudad de México sobre Población y Desarrollo. Proyecto presentado por 29 estados. E/CONF.76/L.4, 13 de agosto, 1984. 
Por otra parte se dio mayor énfasis al reconocimiento de la diversidad y complejidad de situaciones demográficas; a las dificultades implícitas en la elaboración de políticas de población integradas a las de desarrollo; a los vínculos que existen entre la paz y el desarrollo; a la función y condición de la mujer; a la mayor protección del niño y a la vida en familia; a la conveniencia de cuantificar las metas demográficas; mayor especificidad sobre las relaciones existentes entre los factores demográficos y variables sociales y económicas; señalamientos más concretos sobre las necesidades de investigación, sobre el papel de los gobiernos nacionales, la función de la cooperación internacional y la necesidad de mayor vigilancia y evaluación.

Por otra parte, el conjunto de recomendaciones aprobadas constituye una de las guías más completas, y éste es precisamente su objetivo, para la elaboración de políticas de población entendiendo a lo demográfico como resultado complejo de redes de relaciones que van desde lo económico hasta lo cultural e ideológico, pasando por las diversas organizaciones en que se finca la estructura de los sistemas sociales y la concepción del Estado.

Pareciera que el conjunto de lo aprobado en la Ciudad de México descansa en la siguiente visión teórica de la población: El monto, el ritmo de crecimiento y la composición por edad y sexo de las poblaciones humanas responden a las transformaciones que se dan en los tres componentes demográficos básicos: la mortalidad, la fecundidad y la movilidad geográfica. Los factores señalados a su vez interactúan conformando una dinámica particular asociada a situaciones económicas, sociales y políticas que obligan a plantear a la población como un elemento íntimamente ligado a los condicionamientos históricos de la situación social de que se trate. La mortalidad, la fecundidad y las migraciones constituyen factores que difieren entre sí por su naturaleza propia y por la manera en que son considerados en términos de los condicionamientos sociales y culturales del desarrollo. Por otra parte, el ritmo del crecimiento, el monto de la población y la estructura por edad y sexo -consecuencias inmediatas de los tres componentes demográficos básicos-, actuán sobre el conjunto de la sociedad e influyen en la determinación de orientaciones y políticas, poniendo en juego la estructura institucional e ideológica de la sociedad. De alli que la población y su estudio implican la consideración de la sociedad en su conjunto, o sea sus determinaciones económicas, sociales y culturales, lo juridico e institucional, lo ideológico y lo político. Conviene para cada fenómeno demográfico señalar algunas de sus características; considerar la manera en que interactuan y finalmente llegar a establecer de qué dependen y sobre qué influyen en el 
proceso de cambio de la sociedad. No obstante, puede considerarse la disminución del crecimiento demográfico sobre todo cuando se está frente a tasas muy elevadas que dificultan el logro de mejores condiciones de vida de la población.

Es precisamente en estos últimos aspectos en donde se acentúa el pragmatismo de las conclusiones y las recomendaciones que orientan la acción futura del Plan de Acción Mundial sobre Población: nadie puede negar que existen relaciones entre la población y el desarrollo, pero tampoco es posible determinar el carácter concreto de la interrelación entre la situación demográfica y el desarrollo socioeco. nómico sobre todo a corto y mediano plazo y es claro para todos que la planificación familiar constituye un logro de la humanidad.

He dejado para el final la mención de reservas para tres recomendaciones de las 88 aprobadas, por el hecho de que constituyen temas sobre los que se seguirá discutiendo en el futuro:

Formularon reservas a la recomendación 3 la India, la RSS de Ucrania y la URSS, señalando que "No pueden aceptar la redacción poco afortunada de la recomendación 3 , que implica una subestimación del papel que el sector estatal está desempeñando en el desarrollo socioeconómico, tal como se refleja en los documentos pertinentes de las Naciones Unidas".

Suecia formuló reservas a la recomendación 18 . El segundo párrafo de su declaración dice: "La delegación de Suecia deplora sobremanera que se haya adoptado una enmienda para suprimir la palabra "ilegal" en la recomendación (18e), lo cual indica que esta Conferencia ha sido incapaz de reconocer la importancia de este problema tan grave. La delegación de Suecia desea reiterar que un paso importante para eliminar el aborto ilegal consiste en proporcionar acceso al aborto legal y seguro a todas las mujeres del mundo".

Y México formuló reservas para la recomendación 88. “(. . .) no corresponde al Secretario General de las Naciones Unidas observar la aplicación de los programas de población que cuentan con asistencia multilateral, ya que sólo puede ser una prerrogativa de los gobiernos. En este sentido el Secretario General únicamente puede observar la aplicación de la asistencia que provean las agencias del sistema de las Naciones Unidas en materia de población a los programas gubernamentales". 


\title{
ANEXO \\ VERSIÓN ABR EVIADA DE LAS RECOMENDACIONES* (R) A LOS ESTA DOS PARA LA ACCIÓN FUTURA**
}

\author{
R AÚL BENÍTEZ ZENTENO
}

A. Desarrollo socioeconómico y población (14Rs).

(R1) Enfoque integrado; (R2) objetivos concretos del 3er. decenio de las N.U. para el desarrollo, tomar en cuenta las tendencias demográficas; (R3) instancia internacional para eliminar barreras al desarrollo (al comercio); hacer frente a necesidades de empleo; abastecimiento alimentario; mejoramiento de la calidad de vida; autosuficiencia; (R4) adopción de políticas demográficas para corregir desequilibrios entre el crecimiento demográfico y necesidades.

B. Función y condición de la mujer (6Rs).

(R5) Capacidad de la mujer para regular su propia fecundidad base para el disfrute de otros derechos; (R6) perfeccionar y proteger los derechos de la mujer, participación libre y sin restricciones en la fuerza de trabajo; (R7) aplazamiento de la procreación para realización personal; (R8) elevar la edad al matrimonio;

* Las recomendaciones se inician haciendo un llamado a los gobiernos (o a los sujetos de la recomendación) para que .. .

** Con base en el Informe de la Conferencia aprobado. Documento E/CONF.76/L.3, 13 de agosto de 1984. Relatora Srita. Mercedes B. Concepción. 
(R9) participación activa de los hombres en la familia, crianza hijos y trabajo doméstico; (R10) eliminación de todas las formas de discriminación a la mujer.

C. Elaboración de políticas demográficas (2Rs).

(R11) Que se refuercen mutuamente las políticas demográficas y las de desarrollo; (R12) destinar recursos para las políticas demográficas.

D. Metas y políticas demográficas (47Rs).

D.1. Crecimiento de la población (1R): posibilidad de fijar metas cuantitativas. (R13)' Cuando se estime que el crecimiento demográfico dificulta el desarrollo, aplicar políticas demográficas en el marco de las políticas de desarrollo.

D.2. a) Mortalidad y morbilidad (12Rs).

(R14) Metas: países con alta mortalidad lograr para el año 2000 una esperanza de vida al nacimiento de 60 años y mortalidad infantil de 70 ; países con mortalidad intermedia lograr para el año 2000 una esperanza de vida al nacimiento de 70 años y una mortalidad infantil de 35; (R15) participación de la comunidad en el mejoramiento sanitario; (R16) salud y su relación con todos los sectores pertinentes de desarrollo nacional y comunitario.

b) (R17) Medidas para determinar causas de morbilidad y mortalidad y llegar a los que no tienen servicios; (R18) no escatimar esfuerzos para reducir morbilidad y mortalidad maternas; (R19) atención primaria de la salud; (R20) apoyo a lactancia natural; (R21) elevar el nivel de educación como fin en sí mismo y de sobrevivencia.

c) (R22) Control de enfermedades infecciosas y contagiosas; (R23) información y salud; (R24) eliminar consecuencias negativas salud de muchas ocupaciones; (R25) ambiente familiar a todos.

D.3. La procreación y la familia. (R26) Urgente: información, enseñanza y medios a nivel universal para ayudar parejas a lograr el número de hijos deseado; (R27) recursos a los servicios de planificación familiar; (R28) mejorar calidad y eficacia de la planificación familiar y vigilar servicios; (R29) educación adecuada a adolescentes; (R30) que todos, parejas e individuos tengan el derecho fundamental de decidir libre y responsablemente el número y espaciamiento de los hijos; (R31) no coerción, no discriminación y compatibles derechos humanos; (R32) traducción de 
objetivos en fecundidad en políticas y medidas operacionales concretas; (R33) metas cuantitativas de fecundidad; (R34) familia y necesidades: apoyo financiero, servicios de bienestar infantil, licencia maternidad y paternidad, asistencia parejas; (R35) para reducir fecundidad deben aplicarse políticas de desarrollo (salud, educación, integración mujer, etcétera).

D.4. Distribución de la población y migración interna. (R36) Prohibiciones en relación con territorios ocupados para traslado de población nativa o transferir población de ocupación; prohibición de traslados en masa o individuales; (R37) políticas de distribución de la población y análisis de costo beneficio para las personas; (R38) consecuencias políticas de las formas de distribución demográfica.

D.5. a) Migración internacional. (R45) Migración internacional y apoyo a derechos humanos; (R46) las políticas de migración internacional deben tomar en cuenta al migrante, a su familia y las consecuencias demográficas; (R47) se debe asignar gran prioridad a la rehabilitación de personas por catástrofes naturales o provocadas por el hombre.

b) Trabajadores migratorios documentados. (R48) Trato igual a migrantes y trabajadores nacionales;(R49) medidas para normalizar la vida familiar; (R50) informar y educar migrantes; (R51) difusión para comprensión situación del trabajador migratorio.

c) Migrante indocumentado. (R52) Todas las medidas para entrada, salida, reducción ingreso o permanencia deben respetar derechos humanos básicos; (R53) tomar en cuenta las pautas establecidas en el Convenio de la OIT.

d) Refugiados. (R54) Cooperación internacional en el marco de las N.U. para evitar nuevas corrientes de refugiados. Se pide a los países se readhieran a los instrumentos internacionales sobre refugiados; (R55) soluciones duraderas a los problemas relativos a los movimientos de refugiados y eliminación de sus causas.

D.6. Estructura de la población. (R56) Tener presentes las consideraciones que dieron lugar a la proclamación del año internacional del niño; (R57) redoblar esfuerzos con la juventud; (R58) reafirmar compromiso de aplicar el Plan de Acción Internacional sobre el Envejecimiento; (R59) 
considerar al planificar las consecuencias de los cambios de las estructuras demográficas.

E. Reunión y análisis de datos (9Rs).

E.1. (R60) Establecer mecanismos permanentes para la reunión, procesamiento y análisis de datos. Asistencia internacional para estos procesos; (R61) vigilancia y evaluación de las tendencias demográficas y sus repercusiones en forma periódica; (R62) publicación desglosada de datos por sexos; (R63) tabular y publicar datos de grupos minoritarios; (R64) perfeccionamiento de censos, encuestas, registros sobre migración; (R65) mejorar censos de población y habitación para 1990; (R66) desarrollo de encuestas integradas para perfeccionar programas nacionales; (R67) salvaguarda de la confidencialidad estadística;(R68) mejorar y publicar oportunamente estadísticas para programas demográficos y de salud.

E.2. Investigación (4Rs).

(R69) Más recursos para investigación en reproducción humana; (R70) prioridad a la investigación de servicios y operación para mejorar servicios de planificación familiar; (R71) apoyo para desarrollar y mantener eficacia de la capacidad de investigación a nivel nacional, regional y mundial; (R72) especial importancia a la investigación sobre la integración de los procesos demográficos y el desarrollo socioeconómico.

E.3. Gestión, capacitación, información, educación y comunicación (4Rs).

(R73) Apoyo a programas de población y a todo tipo de capacitación; (R74) búsqueda de nuevos métodos para comprensión de los factores demográficos y lograr participación activa de la población; (R75) formación expertos en políticas demográficas, programas, etc.; (R76) uso por parte de los gobiernos de la mejor información y análisis demográficos.

\section{RECOMENDACIONES PARA LA EJECUCIÓN(12Rs)}

A. Papel de los gobiernos nacionales. (R77) Alta prioridad al logro de autosuficiencia en la gestión de sus programas de población; (R78) mayor uso de cooperación técnica entre los países y las regiones. 
B. Función de la cooperación internacional. (R79) La comunidad internacional debe adoptar medidas internacionales adecuadas para desempeñar un importante papel en la ejecución del Plan de Acción Mundial sobre Población; (R80) se insta al sistema de N.U. y países donantes a prestar asistencia a los gobiernos que lo soliciten; (R81) la comunidad internacional debe hacer hincapié en los aspectos de investigación y acción; institucionalización de la integración de la planificación demográfica en el proceso de desarrollo; el mejoramiento de la condición juridica y social de la mujer; la investigación biomédica y en ciencias sociales; reunión y análisis de datos; identificación de programas exitosos; aplicación de sistemas de evaluación y vigilancia; promoción de intercambios; enseñanza y capacitación;(R82) se insta a los gobiernos a aumentar la cuantía de la asistencia para lograr los objetivos del 3er. decenio de las N.U. para el desarrollo; (R83) debe reforzarse aún más el Fondo de las N.U. para Actividades en Población; (R84) se invita a las organizaciones nacionales no gubernamentales a que continúen su labor precursora; (R85) se invita a los parlamentarios, a los medios de información a que se cobre conciencia de los problemas de población y desarrollo; (R86) se insta a parlamentarios y a quienes participen en actividades públicas a adoptar un criterio eficaz e integrado para la solución de los problemas de la población y el desarrollo; (R87) se insta a los órganos de gobierno de las N.U. a que incluyan las cuestiones de población entre sus principales prioridades.

C. Vigilancia, examen y evaluación. (R88) El Secretario General de las Naciones Unidas, como se indica en el Plan, debe seguir encargándose de la vigilancia de las tendencias y políticas demográficas, así como de los programas de población multilaterales apoyados por las Naciones Unidas, a través de los acuerdos apropiados. El próximo examen y evaluación global de los progresos en la ejecución del Plan de Acción Mundial sobre Población será en 1989 (textual). 